\title{
Laboreal
}

Volume $13 \mathrm{~N}^{\circ} 1$ | 2017

Varia

\section{Los espacios de debate y el poder de actuar en la construcción de la seguridad de las organizaciones}

Espaços de debate e poder de agir na construção da segurança das organizações Les espaces de débat et le pouvoir d'agir dans la construction de la sécurité des organisations

Debate spaces and the power to act upon the construction of safety in the organisations

\section{Raoni Rocha}

\section{OpenEdition}

\section{Journals}

Edición electrónica

URL: http://journals.openedition.org/laboreal/1989

DOI: 10.4000/laboreal.1989

ISSN: 1646-5237

\section{Editor}

Universidade do Porto

\section{Referencia electrónica}

Raoni Rocha, «Los espacios de debate y el poder de actuar en la construcción de la seguridad de las organizaciones », Laboreal [En línea], Volume $13 \mathrm{~N}^{0} 1$ | 2017, Publicado el 01 julio 2017, consultado el 24 septiembre 2020. URL : http://journals.openedition.org/laboreal/1989 ; DOI : https://doi.org/ 10.4000/laboreal. 1989

Este documento fue generado automáticamente el 24 septiembre 2020.

\section{c) (1) 8}

Laboreal está licenciado com uma Licença Creative Commons - Atribuição-NãoComercial 4.0 Internacional. 


\section{Los espacios de debate y el poder de actuar en la construcción de la seguridad de las organizaciones}

Espaços de debate e poder de agir na construção da segurança das organizações Les espaces de débat et le pouvoir d'agir dans la construction de la sécurité des organisations

Debate spaces and the power to act upon the construction of safety in the organisations

\section{Raoni Rocha}

\section{REFERENCIA}

Rocha, R. (2014). Du silence organisationnel au débat structuré sur le travail : les effets sur la sécurité et sur l'organisation. Thèse de doctorat préparée à l'École doctorale SP2 'Sociéte, Politique, Santé Publique', spécialité Ergonomie, Université de Bordeaux.

\section{NOTA DEL EDITOR}

http://dx.doi.org/10.15667/laborealxiii0117rr

Manuscrito recibido en: Diciembre/2016

Aceptado tras peritaje: Marzo/2017

Júri da tese: Francisco Duarte (Presidente); François Daniellou (Orientador) e Vanina Mollo (Co-orientadora); Pierre Falzon e Benoit Journe (Arguentes). 


\section{Introducción}

1 Los modelos y herramientas actuales de gestión de la seguridad en las empresas son antiguos y se revelan, a menudo, ineficaces para garantizar la seguridad de los sistemas. Diferentes investigaciones subrayan la importancia de restablecer el colectivo de trabajo, de implantar prácticas reflexivas entre los trabajadores y de poner en tela de juicio el trabajo real (Daniellou, Simard, \& Boissières, 2010). Sin embargo, son limitadas las investigaciones que, de hecho, lograron desarrollar espacios de debate sobre el trabajo real y no se conocen muchas contribuciones efectivas de un enfoque de ese tipo ni cuales las condiciones que permitirían su desarrollo. Por consiguiente, se desarrolló una investigación doctoral en una empresa de distribución de energía eléctrica con un sistema de control estricto y sanciones en caso de incumplimiento de las normas. La investigación puso en práctica espacios de debate entre trabajadores de distintos rangos. El objetivo de este resumen de tesis es, pues, enseñar cuales son las contribuciones sistémicas y las condiciones para el desarrollo de un enfoque que busque hacer del debate sobre el trabajo una herramienta que permita el desarrollo de una gestión de la seguridad eficaz.

\section{Del silencio organizacional a los espacios de debate sobre el trabajo}

2 El "silencio organizacional" surge en las empresas cuando "la opción dominante al interior de varias organizaciones para los empleados es guardar para sí mismos sus opiniones y preocupaciones con respecto a los problemas organizacionales" (Morrison \& Milliken, 2000, p. 707). Esta definición muestra que el silencio del empleado es el resultado de una decisión deliberada y que la información omitida puede devenir crítica para el sistema. Dos razones desalientan cualquier forma de expresión (Rocha, Mollo \& Daniellou, 2015): el miedo de repercusiones negativas y la percepción de una débil comunicación de informaciones con respecto al campo. Por temor a la forma en la que los gestores o pares recibirán el mensaje, y por estar convencido de que sus jefes no están muy disponibles para escucharlo o para tratar algunos de los problemas del campo, el individuo no revela las informaciones, ya que, en cualquier caso, piensa que eso no producirá ningún resultado práctico (Dejours, 1998).

Con el objetivo de romper el silencio organizacional, Detchessahar (2001) desarrolla la "teoría de los espacios de discusión", preconizando la discusión sobre el trabajo en el plan de acción, de forma regular y segura, coordinada por un gestor que, en función del tema, puede o no tener una relación jerárquica con los miembros del equipo. La idea de la discusión sobre el trabajo no puede, sin embargo, disociarse del poder de actuar [1] de los participantes de estos espacios en el tratamiento de las situaciones discutidas. Esta reflexión evoca el principio de subsidiariedad, que trabaja con las nociones de "poder" y "autonomía" de los grupos sociales. Este concepto define los principios de distribución de poder en una comunidad y su principal interés es el hecho de pensar, a la vez, la libertad y la autoridad de los individuos (Millon-Delsol, 1993). En el mundo empresarial, el concepto de subsidiariedad se traduce por el hecho de que un nivel de gestión superior no debe ejercer las funciones que un nivel jerárquico inferior puede realizar de manera eficiente. Al mismo tiempo, los niveles inferiores deben tener apoyo en la coordinación entre sus actividades y las de la organización sistémica (Melé, 2005). 
El principio de subsidiariedad remite, pues, a la "investigación permanente del nivel más pertinente para la acción" (Petit, Dugué, \& Daniellou, 2011, p. 404) a partir de la articulación y del ajuste de los diferentes colectivos de la organización.

\section{Métodos de campo y del análisis de los datos}

4 Con el objetivo de crear espacios de debate para gestionar temas de seguridad, se desarrolló una investigación en una empresa francesa de distribución de energía eléctrica. El objetivo principal de la investigación fue desarrollar espacios de debate sobre el trabajo (EDT) interconectados por el principio de subsidiariedad como forma de romper el silencio organizacional. Para ello, se formaron dos comités: un Comité Científico, compuesto por tres investigadores (el estudiante de doctorado, con su director y codirector); y un Comité Ejecutivo, compuesto por los tres investigadores y cuatro representantes de la Dirección de la empresa. Ambos comités mantenían un diálogo constante con el Comité Directivo (Codir) de la empresa, compuesto por los miembros de la Junta Directiva.

El método de recopilación de datos de esta investigación tuvo dos grandes fases. La primera fue una fase de experimentación de los EDT, con el objetivo de desarrollar el debate de las situaciones de campo relacionadas con la seguridad y contó con las subfases "análisis del trabajo", "tratamiento de las situaciones de acción características - SAC" y "experimentación del dispositivo de debate". La segunda fue una fase de perennización / generalización de los EDT y de organización de la subsidiariedad que relacionó los espacios de debate locales con los niveles superiores de la empresa. En esta fase se llevaron a cabo formaciones y el seguimiento de las acciones de los gestores con respecto a la implantación de los dispositivos de debate. Reuniones periódicas entre los comités, así como presentaciones de la investigación al Codir, se llevaron a cabo constantemente durante el desarrollo del proyecto. La siguiente figura ilustra la metodología utilizada.

Figura 1: Metodología de la investigación

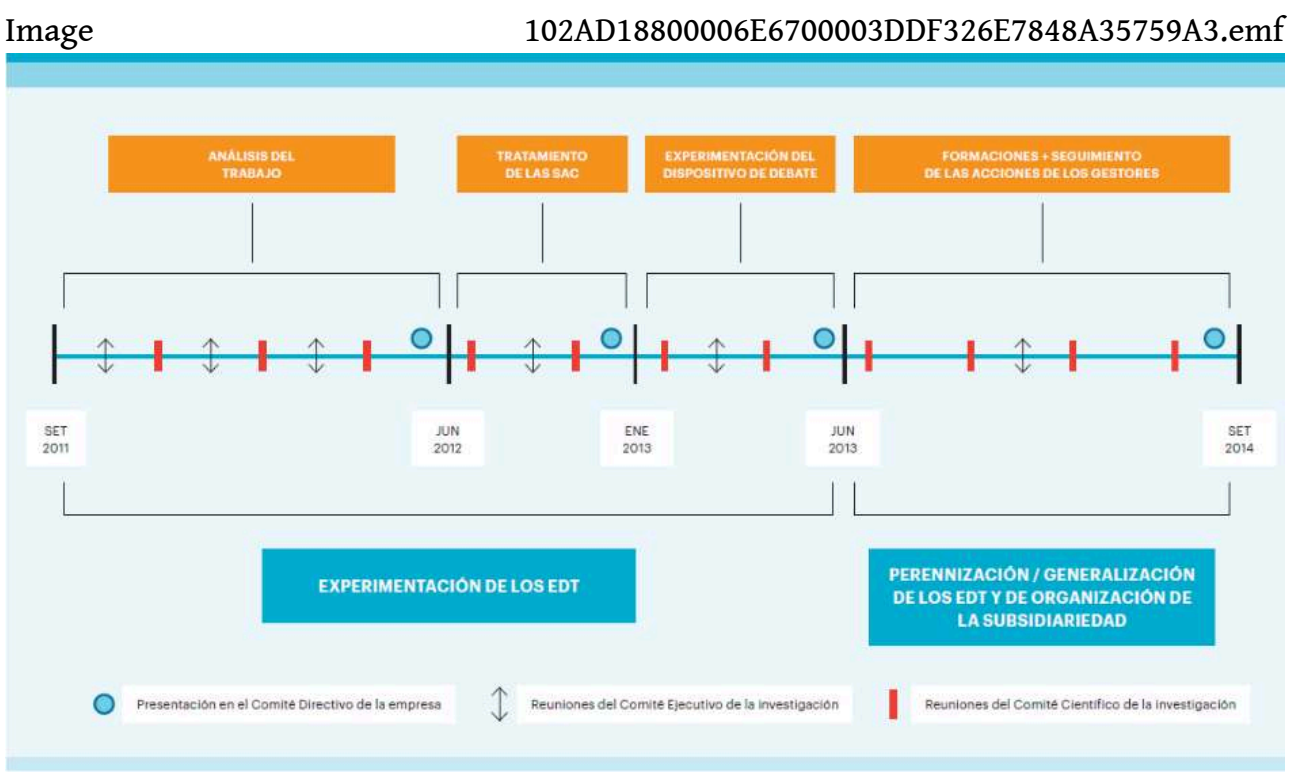


Se realizó un análisis del contenido de los debates en la unidad piloto: el la subfase de tratamiento de las SAC y de experimentación del dispositivo de debate, se grabaron y trascribieron las reuniones. El material transcrito se analizó de forma cualitativa (tratamiento temático de acuerdo con las grandes variables del debate) y cuantitativa, con la ayuda del Actogram Kronos (Kerguelen, 2003), un software de tratamiento de datos de observación cronológica de variables predeterminadas. Los gráficos producidos por este software permitieron obtener una "fotografía" de la sesión completa, que muestra la evolución de todas las variables codificadas en el mismo gráfico y que también nos permitió observar la variación de los datos entre las sesiones registradas [2].

\section{Resultados: la producción de un debate subsidiario}

7 En cuatro unidades de la empresa se co-construyó un sistema de exposición y tratamiento de las anomalías de campo que permitió compartir y tratar localmente estas anomalías. La presente tesis se centró en los resultados de la unidad piloto. En esta unidad, se implementó un dispositivo local de debate, en el que los electricistas tomaban fotografías de los eventos significativos del campo y las pasaban al supervisor en el debriefing. Tras la selección del supervisor, las fotografías pasaban por un tratamiento colectivo (figura siguiente).

Figura 2: Dispositivo local de debate sobre el trabajo

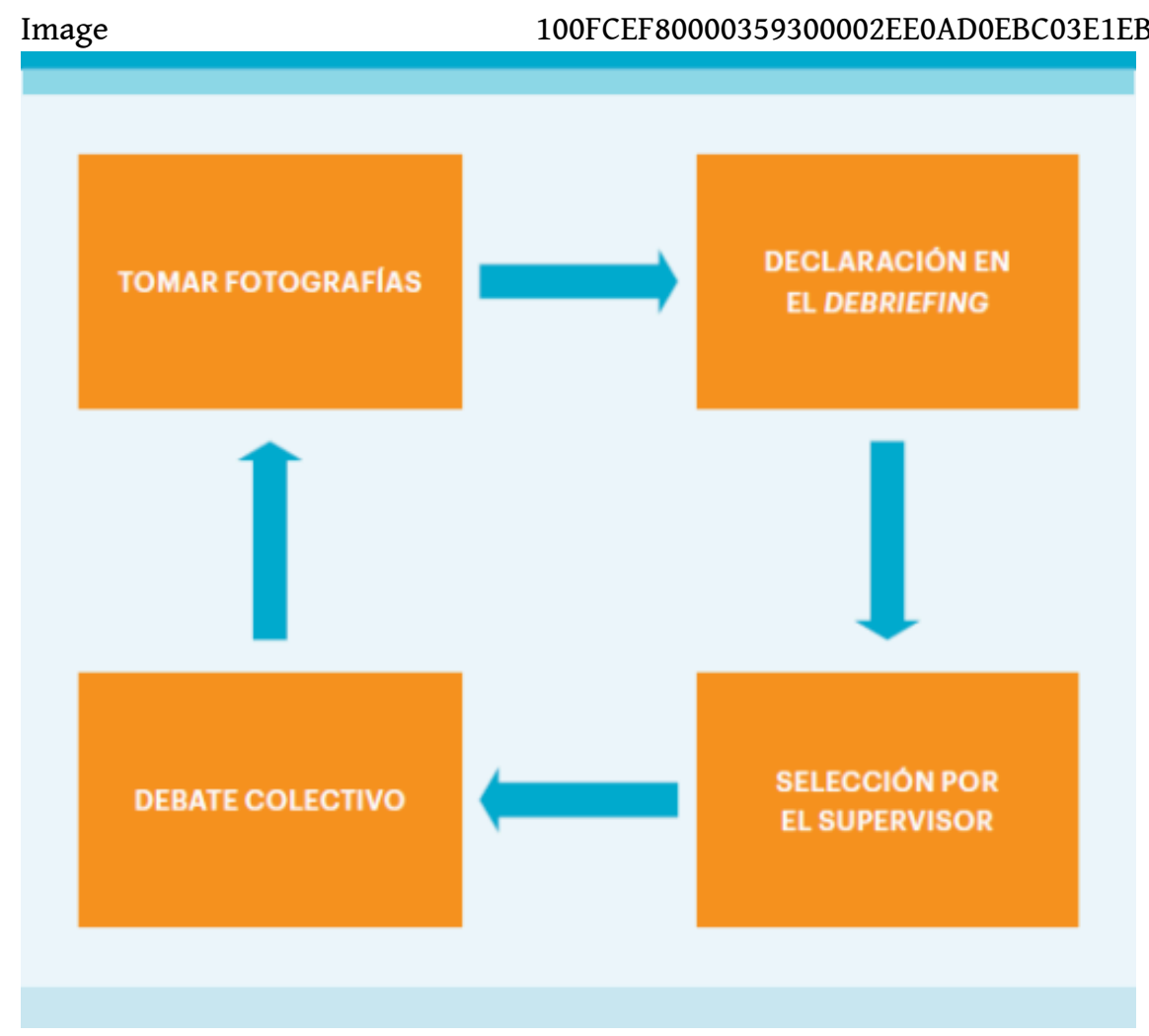

8 Este dispositivo proporcionó un aumento de la exposición y del tratamiento de situaciones de riesgo; permitió también una apropiación progresiva de los participantes, haciéndoles hablar más y más sobre el trabajo, e hizo posible el aprendizaje mutuo entre los electricistas. Además, hizo contribuciones en la 
construcción de la salud y en el desarrollo del colectivo local de trabajo (Rocha, Mollo y Daniellou, 2015).

En la medida en que el dispositivo local funcionaba, se observó que los trabajadores tenían condiciones para tratar a nivel local varias de las situaciones discutidas (fusibles quemados, cortacircuitos dañados, ausencia de tornillos o de cableado en algunos de los equipo, etc.). Sin embargo, este dispositivo también acarreó algunas dificultades, sobre todo cuando el grupo no tenía condiciones para tratar todas las situaciones discutidas. Había, por lo tanto, la necesidad de exponer este tipo de situaciones a otro nivel que pudiera tratarlas. Desde esta perspectiva, se decidió, de común acuerdo con el sector de prevención de la empresa (involucrado en el proyecto desde sus inicios), que las situaciones no tratadas en la unidad piloto se comunicarían al sector de prevención a través de reuniones mensuales del Comité de Prevención-Seguridad (CPS). En los casos en que el sector de la prevención no tenía recursos para tratar algunas situaciones, estas se transmitían a los directivos de la empresa durante las reuniones trimestrales del Codir, que contaban con la participación del responsable por la prevención. La siguiente figura muestra la evolución del dispositivo sistémico construido con los diferentes trabajadores y de niveles de la empresa.

Figura 3: Dispositivo de debate sistémico sobre el trabajo.

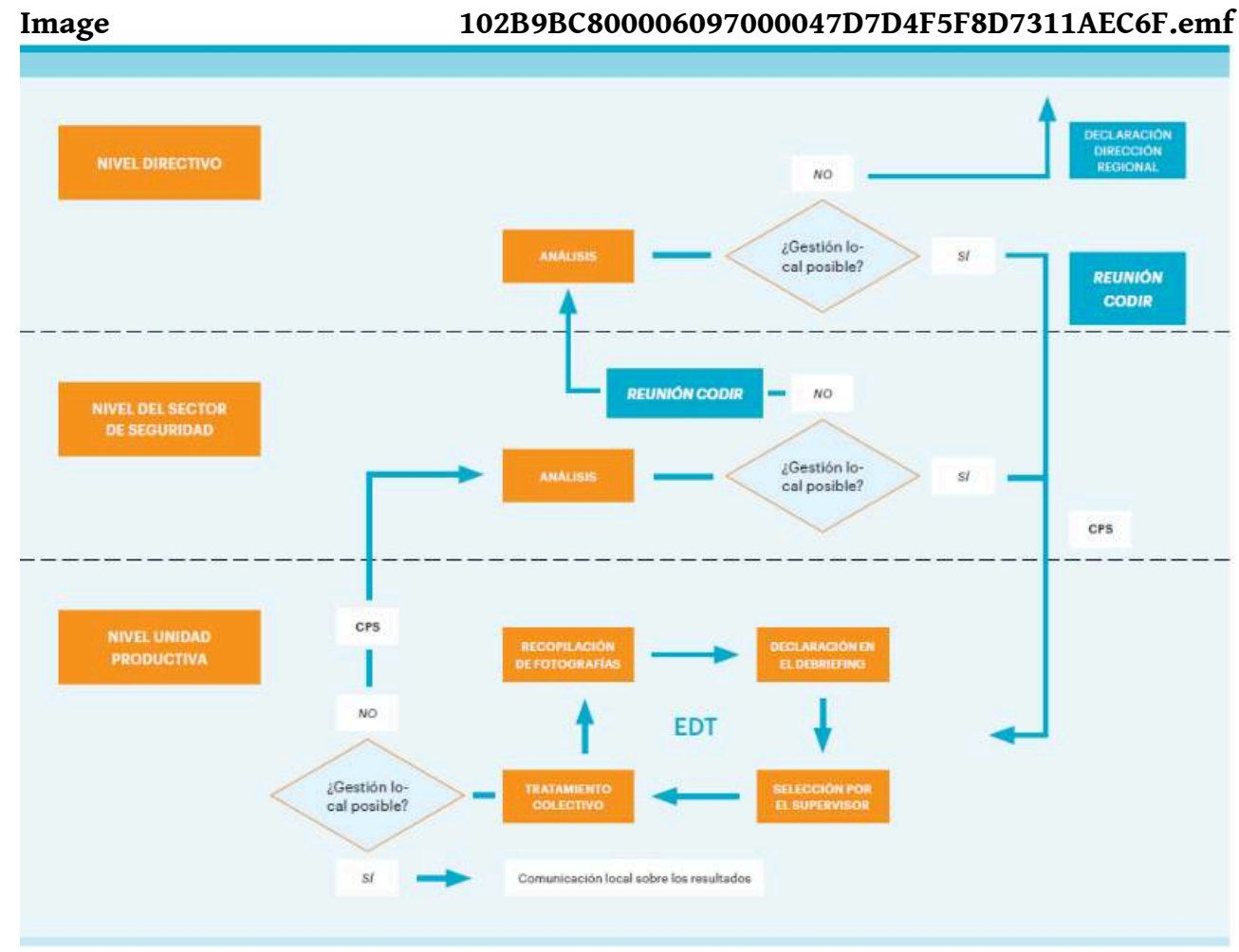

\section{Contribuciones sistémicas y condiciones del debate estructurado sobre el trabajo}

El desarrollo del debate local regido por el principio de subsidiariedad permitió, primeramente, el desarrollo de la innovación en la empresa. A título de ejemplo, un electricista relata una situación de mejora en la bolsa de herramientas, tras haber visto 
una herramienta caer de esa bolsa, en una intervención en un poste. Este ejemplo muestra no sólo la acción del debate sobre la seguridad (ya que la modificación de la bolsa evita accidentes potencialmente graves), pero también genera situaciones de innovación de materiales previamente existentes. Posteriormente, el debate sobre el trabajo pudo crear condiciones de reconexión de la gestión de la empresa con la realidad del trabajo, tanto por parte de la unidad piloto, como por parte de los gestores de otros niveles jerárquicos. El supervisor coordina, sugiere y compara los puntos de vista. Accede a la realidad del trabajo de los electricistas, a los ajustes efectuados y a las modalidades de tratamiento de las situaciones discutidas, lo que permite su acercamiento a una realidad que antes no resultaba muy clara. Por último, el debate estructurado sobre el trabajo permitió la anticipación de riesgos y la prevención de accidentes a través del desarrollo de una dinámica de aprendizaje cuotidiano y del intercambio de conocimientos técnicos entre los participantes. El intercambio y la gestión de las situaciones vividas permitieron, en algunos momentos, aprender del grupo, y en otros, enseñar al grupo: "los temas que discutimos aquí, nunca había visto en mi formación", "ahora lo entiendo" y "no lo sabía" fueron algunas de las declaraciones expresadas por los electricistas.

11 También se pueden establecer algunas condiciones para que se desarrolle el debate sobre el trabajo estructurado por el principio de subsidiariedad.

12 Capacidad de la Dirección para desafiar sus propios límites: si el espacio de debate evidencia la actividad real, él también permite hacer visibles las vulnerabilidades de la empresa y sus causas. Desde este punto de vista, la administración puede no querer conocer sus vulnerabilidades $\mathrm{y}$, por eso, impedir esta práctica. Aceptar escuchar opiniones diferentes a la suya es fundamental para que los errores y las infracciones de la prescripción puedan expresarse sin que la Dirección utilice su poder coercitivo para interrumpir el proceso. La cuestión es, entonces, la propia capacidad de conocer su actividad y tratar las vulnerabilidades.

Un gerente que garantice y anime el debate local: el supervisor tenía un papel central en la efectividad de los espacios de debate. Era a la vez el garante y el animador del debate, destinando de forma sistemática una parte de las reuniones semanales para la discusión colectiva en torno a las fotos presentadas. Fomentaba el debate, organizaba el tratamiento de las situaciones de riesgo discutidas y establecía el enlace entre las situaciones no tratadas a nivel local y el sector de la prevención de la empresa. Por último, comunicaba de forma sistemática con el grupo sobre el tratamiento de las situaciones analizadas. Este raciocinio puede extenderse también a los gestores intermedios de la empresa. Aseguraron tanto la articulación entre el contenido de las situaciones trabajadas en los diferentes niveles jerárquicos con los interlocutores de niveles superiores e inferiores, como el período de debate en algunas de sus reuniones.

Un método co-construido: los espacios de debate han sido objeto de adaptaciones particulares para responder a las especificidades de las unidades trabajadas. En la unidad piloto, el debate se hizo a través de fotografías (en otras unidades se llevó a cabo a través de vídeos o de informes de casos), los casos se comunicaban al supervisor (en otras unidades se comunicaban a los gestores operacionales) y los grupos estaban formados por electricistas y jefes directos (en otras unidades la reunión tuvo lugar con jefes indirectos o con trabajadores de otros sectores). El dispositivo sistémico siguió la misma línea. Se decidió, con el responsable por la prevención, que se utilizaría una parte de la reunión del CPS. En otras unidades, las situaciones no tratadas a nivel local 
se comunicaban directamente a los gestores intermedios o al sector de los recursos humanos. Esta co-construcción fue primordial, ya que permitió integrar un nuevo método en una estructura organizacional existente, sin la necesidad de reuniones adicionales $\mathrm{y}$, por lo tanto, sin un coste suplementario para los trabajadores involucrados en el proyecto.

Un debate basado en la confrontación con lo real, con un colectivo regular y con posibilidad de acción: todo el proceso de debate se ha centrado siempre en el trabajo real de los sujetos (lo que, de hecho, hacen), mediado por fotografías de situaciones del campo. Esta es una de las condiciones fundamentales del debate: debe centrarse en la actividad laboral. Para ello, debe estar apoyado por materiales de suporte, tales como fotografías, videos o relatos, para que no haya digresiones con respecto al tema central (por ejemplo, las cuestiones salariales). Al mismo tiempo, el debate debe realizarse dentro de una lógica de confrontación de ideas y con una presencia constante de sus miembros. Fue en la confrontación de conocimientos de los sujetos regularmente presentes que se observaron las situaciones más ricas en términos de aprendizaje. Por último, es necesario que los participantes tengan la posibilidad de actuar sobre las situaciones discutidas. De lo contrario, como expresa uno de los participantes, "el debate sobre el trabajo ciertamente no tendrá larga vida si sólo se limita a la retórica y no puede ser tratado".

Proporcionar recursos a los niveles inferiores: es necesario que los participantes del debate tengan la posibilidad de actuar sobre las situaciones discutidas. En cada situación presentada al sector de prevención o al Codir, la reflexión construida estuvo presente no sólo en el tratamiento de la situación, pero sobre todo en la oferta de recursos al nivel inferior para que pudiera tratar una situación de ese tipo. Cuando el responsable por la prevención se ocupa de la modificación de una norma para adaptarla a la realidad o cuando la Dirección realiza un trabajo en colaboración con los proveedores de servicios con el fin de impedir la instalación de equipos que puedan causar problemas a los electricistas, no es solo la gestión de estas situaciones que está en juego. En tales casos, los trabajadores locales ganan autonomía y poder de actuar para desarrollar sus acciones frente a situaciones similares en el futuro. Por consiguiente, el objetivo del debate estructurado sobre el trabajo no es aumentar el número de situaciones comunicadas a otros niveles (y correr el riesgo de saturar sus capacidades de tratamiento), sino comunicar lo mínimo posible, a fin de que ese mínimo se convierta en lo esencial y pueda tener una solución definitiva.

\section{Conclusión}

17 Esta investigación intentó desarrollar el debate sobre el trabajo, demostrar sus contribuciones y reflexionar sobre sus condiciones de implantación. El desarrollo del debate estructurado sobre el trabajo (es decir, la construcción de espacios de debate organizados por el principio de subsidiariedad) posibilitó el acceso a estos problemas y su tratamiento de forma duradera. Las antiguas cadenas de mando y líneas de comunicación verticales se transformaron en interacción y participación horizontal en las unidades implicadas. El supervisor de la unidad comenzó a actuar como un coordinador y no un controlador. Los individuos dejaron de ser considerados individualmente responsables por los problemas, ya que el producto del debate era compartido. Así, el debate estructurado sobre el trabajo puso en duda los métodos 
clásicos de gestión de seguridad, basados en una gestión directiva, alejada del campo e inmersa en una producción excesiva de procedimientos de seguridad. Siempre y cuando se respeten determinadas condiciones, el debate y su articulación con los niveles jerárquicos pueden permitir una gestión cotidiana de las situaciones problemáticas y superar el perímetro clásico de la seguridad para ir hacia una organización capaz de identificar, anticipar y gestionar los problemas de forma rutinera.

\section{BIBLIOGRAFÍA}

Clot, Y. (2010). Trabalho e poder de agir. Belo Horizonte: Fabrefactum.

Daniellou, F., Simard, M., \& Boissières, I. (2010). Fatores Humanos e Organizacionais da Segurança Industrial. (Traduzido do original Facteurs humains et organisationnels de la sécurité industrielle por Rocha, R., Duarte, F., e Lima, F.). Toulouse: ICSI. Disponível no link: http://www.FonCSI.org/ fr/cahiers/

Dejours, C. (1998). Souffrance en France. La banalisation de l'injustice sociale. Paris : Seuil.

Detchessahar, M. (2001). Quand discuter, c'est produire... Pour une théorie de l'espace de discussion en situation de gestion. Revue française de gestion, 132, 32-43.

Kerguelen, A. (2003). Actogram Kronos pour Windows, Editions Octarès : Toulouse.

Melé, D. (2005). Exploring the Principle of Subsidiarity in Organisational Forms. Journal of Business Ethics, 60, 3, 293-305. https://doi.org/10.1007/s10551-005-0136-1

Millon-Delsol, C. (1993). Le Principe de subsidiarité, Paris, PUF (coll. « Que sais-je ? »).

Morisson, E., \& Miliken, F. (2000). Organizational Silence: a barrier to change and development in a pluralistic world. The Academy of Management Review, 25, 4, 706-725. https://doi.org/10.5465/ AMR.2000.3707697

Petit, J., Dugué, B., \& Daniellou, F. (2011). L'intervention ergonomique sur les risques psychosociaux dans les organizações : enjeux théoriques et méthodologiques. Le travail humain, 74, 4, 391-409.

Rocha, R., Mollo, V., \& Daniellou, F. (2015). Work debate spaces: a tool for developing a participatory safety management. Applied Ergonomics, 46, 107-114. https://doi.org/10.1016/ j.apergo.2014.07.012

\section{NOTAS}

1. La expresión "poder de actuar" se utiliza aquí como la capacidad de los trabajadores tienen para resolver los problemas que se les presentan en la situación de trabajo y, consiguientemente, las condiciones ofrecidas por la gestión para que eso sea posible. Esta definición está en línea con la advertencia que Yves Clot (2010) hace en su libro "El trabajo y el poder de actuar" (páginas 14 y 15), advirtiendo que "desde que las nociones de potencia de acción, poder de actuar y poderes de la acción se introdujeron en el campo de la psicología del trabajo (...), se convirtieron en objeto de 
múltiples usos (...)" y que "esta diversidad es de gran interés. Una vez reconocida, ella invita a una intensa discusión, hecha posible hoy en día. Este debate también es necesario para evitar, sin duda, que la expresión sólo se convierta en un acceso fácil a la acción".

2. Para más detalles sobre la metodología, consultar: Rocha, R. (2014). Du silence organisationnel au débat structuré sur le travail: les effets sur la sécurité et sur l'organisation. Tesis (Doctorado en Ergonomía) - Université de Bordeaux, Bordeaux.

\section{AUTOR}

\section{RAONI ROCHA}

Engenharia de Saúde e Segurança

Universidade Federal de Itajubá, Campus Itabira, Rua Irmã Ivone Drumond, 200 - Distrito

Industrial II, Itabira - MG, CEP 35903-087 Brasil

raoni@unifei.edu.br 